\title{
MEDIDAS DE PREVENÇÃO DA COVID-19 EM PESSOAS QUE VIVEM COM DIABETES MELLITUS
}

\section{COVID-19 PREVENTION MEASURES IN PEOPLE LIVING WITH DIABETES MELLITUS}

\author{
Álef Lucas Dantas de Araújo Silva ${ }^{1}$ * Lucas David Maia Matias ${ }^{2}$ *Jucicleia Maiara da Silva \\ Freitas $^{3}$ José Carlos Nascimento de Oliveira ${ }^{4} *$ Lidiane Lima de Andrade $^{5}$
}

\section{RESUMO}

Objetivo: Investigar as evidências científicas sobre as medidas de prevenção da Covid-19 em pessoas que vivem com diabetes mellitus. Método: Revisão integrativa da literatura, realizada no período de 20 de junho a 20 de julho de 2020 , baseada na seguinte questão norteadora: quais medidas de prevenção da Covid-19 em pessoas que vivem com diabetes? Foram utilizadas as bases de dados: Medline/Pubmed, LILACS e CINAHL, cruzando-se os descritores: "diabetes mellitus" AND "coronavirus infections" e "diabetes mellitus" AND “covid-19". Incluíram-se estudos experimentais, quase experimentais, observacionais, de reflexão, relatos de experiência, cartas ao editor, revisões sistemáticas, metanálises e integrativas de literatura; e estudos publicados entre dezembro de 2019 a julho de 2020. Excluíram-se pesquisas que não responderam à questão de pesquisa. Resultados: Os dados permitiram a construção de duas categorias, sendo elas: prevenção geral e prevenção específica. Na primeira, foram sugeridos os cuidados: lavar as mãos ou utilizar álcool a 70\%, adotar etiqueta respiratória ao tossir ou espirrar, manter distância mínima de um metro de outras pessoas, usar máscaras descartáveis ou de tecido e preservar bem estar psicológico e emocional. Na segunda, pontuou-se: atenção para a ingesta hídrica, melhorar ingesta de nutrientes, manutenção da terapia oral e insulinoterapia, controlar o índice glicêmico, realizar atividade física, abandonar o tabagismo, procurar acompanhamento médico por telessaúde e buscar vacinação contra outras doenças respiratórias. Conclusão: observa-se a relevância dos achados, visto que nortearão as práticas de prevenção e controle, instrumentalizando um cuidado baseado em fundamentos teórico-científicos, a fim de minimizar os impactos da Covid-19 na vida de usuários diabéticos.

Palavras-chave: Diabetes Mellitus; Infecções por Coronavirus; Prevenção e Controle.

\begin{abstract}
Objective: To investigate the scientific evidence on Covid-19 prevention measures in people living with diabetes mellitus. Method: Integrative literature review, carried out from June 20 to July 20, 2020, based on the following guiding question: what measures to prevent Covid-19 in people living with diabetes? The following databases were used: Medline / Pubmed, LILACS and CINAHL, crossing the descriptors: "diabetes mellitus" AND "coronavirus infections" and "diabetes mellitus" AND “covid-19". Experimental, quasi-experimental, observational, reflection studies, experience reports, letters to the editor, systematic reviews, meta-analyzes and integrative literature were included; and studies published between December 2019 and July 2020. Research that did not answer the research question was excluded. Results: The data allowed the construction of two categories, namely: general prevention and specific prevention. In the first, the following precautions were suggested: washing hands or using $70 \%$ alcohol, adopting respiratory etiquette when coughing or sneezing, maintaining a minimum distance of one meter from other people, wearing disposable or fabric masks and preserving psychological and emotional well-being. In the second, the following points were given: attention to water intake, improving nutrient intake, maintenance of oral therapy and insulin therapy, controlling the glycemic index, performing physical activity, quitting smoking, seeking medical advice by telehealth and seeking vaccination against other respiratory diseases. Conclusion: the relevance of the findings is observed, as they will guide prevention and control practices, providing care based on theoretical and scientific foundations, in order to minimize the impacts of Covid-19 on the lives of diabetic users.
\end{abstract}

Keywords: Diabetes Mellitus; Coronavirus Infections; Prevention and Control.

\footnotetext{
${ }^{1}$ Bacharelando em Enfermagem pela Universidade Federal de Campina Grande. Cuité - PB, Brasil.

${ }^{2}$ Bacharelando em Enfermagem pela Universidade Federal de Campina Grande. Cuité - PB, Brasil.

${ }^{3}$ Enfermeira. Especialista em Saúde da Família e Comunidade pela Secretaria Municipal de Saúde de João Pessoa-PB, Brasil.

${ }^{4}$ Bacharelando em Enfermagem pela Universidade Federal de Campina Grande. Cuité - PB, Brasil.

${ }_{5}^{5}$ Enfermeira. Doutora em Enfermagem. Docente do Curso de Enfermagem da Universidade Federal de Campina Grande. Cuité - PB, Brasil. E-mail: lidiane.lima@ufcg.edu.br.
} 


\section{ARTIGO}

\section{INTRODUÇÃO}

A pandemia causada pela coronavirus disease-19

(Covid-19) representa uma emergência de saúde pública de importância internacional. A Covid-19 se apresenta de forma leve na maioria dos pacientes infectados, mas cerca de 15\% precisam hospitalização, e 5\% desenvolvem doença grave ${ }^{(1)}$. Pessoas acometidas pela forma grave podem desenvolver a síndrome do desconforto respiratório agudo, com alta probabilidade de internação em terapia intensiva, como também, podem evoluir para o óbito ${ }^{(2)}$.

Os fatores de risco para pior prognóstico da doença são idade avançada e presença de comorbidades, sendo mais frequentes hipertensão, diabetes mellitus, doença cardiovascular e/ou pulmonar prévia $^{(1)}$. Desta forma, pessoas que vivem com diabetes mellitus tipo 1 ou tipo 2, uma vez infectadas, podem apresentar complicações mais graves, sobretudo, indivíduos com idade superior a 60 anos, com glicemia instável, e que apresentem outras enfermidades concomitantes, como hipertensão arterial sistêmica e obesidade ${ }^{(3)}$.

Ratificando esse dado, estudo aponta que a hiperglicemia e o diagnóstico de diabetes mellitus são preditores para mortalidade em pacientes com Covid19. Esse achado pode ser devido a esses pacientes terem um estado de inflamação metabólica que os predispõe a uma liberação aumentada de citocinas ${ }^{(4)}$.

Em adição, vale destacar que o diabetes consiste em uma das principais causas de morbimortalidade em todo o mundo, e essa condição está associada a várias complicações macrovasculares e microvasculares, o que afeta a sobrevida geral do paciente ${ }^{(5)}$. Assim, emerge-se uma nova conjuntura com a interação entre duas pandemias, o que representa desafios em diferentes áreas, como a saúde, a econômica, a ambiental, a social e a emocional.

Desta maneira, reduzir a exposição ao vírus é necessário para controlar/retardar a propagação da doença e os impactos negativos, como o aumento da mortalidade ${ }^{(6)}$. Portanto, é preciso verificar a produção científica da literatura nacional e internacional acerca de medidas preconizadas para a prevenção da Covid-19, sejam em âmbito de cuidados gerais, ou até mesmo, no que diz respeito a cuidados específicos com usuários diabéticos, no sentido de promover a prevenção da doença em um grupo de risco.

Os resultados dessa investigação, 


\section{ARTIGO}

facilitarão o levantamento de futuras intervenções, o que permitirá aos indivíduos envolvidos, a aquisição de experiência para o enfrentamento da pandemia e o manejo de sua condição de saúde.

Portanto, delineou-se como questão de pesquisa, quais medidas de prevenção da Covid-19 em pessoas que vivem com diabetes?

\section{OBJETIVOS}

Investigar as evidências científicas sobre as medidas de prevenção da Covid-19 em pessoas que vivem com diabetes mellitus.

\section{METODOS}

Trata-se de uma revisão integrativa de literatura realizada conforme as seguintes etapas: 1) elaboração da questão de pesquisa; 2) busca na literatura; 3) avaliação dos dados; 4) análise dos dados - divisão, exposição e comparação; e 5) apresentação dos $\operatorname{resultados}^{(7)}$.

A busca na literatura ocorreu no período de 20 de junho a 20 de julho de 2020. A questão de pesquisa foi construída embasada na estratégia PICo (P- população: pessoas que vivem com diabetes mellitus; IInteresse: prevenção da Covid-19; CoContexto: interação entre duas pandemias), o que resultou na seguinte questão norteadora: quais medidas de prevenção da Covid-19 em pessoas que vivem com diabetes?

As bases de dados utilizadas foram: Literatura Latino-Americana e do Caribe em Ciências da Saúde (Lilacs), Medical Literature Analysis and Retrieval System Online (Medline/Pubmed) e Cumulative Index of Nursing and Allied Health Literatura (Cinahl).

Os descritores utilizados foram: diabetes mellitus, infecções por coronavírus (coronavirus infections) e covid-19, como palavra sinônima, todos consultados no Descritores em Ciências da Saúde (DeCS) e no Medical Subject Headings (MeSH). Foi realizado o cruzamento em pares, utilizando o operador booleano "AND”, a saber: "diabetes mellitus" AND "coronavirus infections" e "diabetes mellitus" AND "covid-19".

Os critérios de inclusão dos estudos foram: serem estudos experimentais, quase experimentais, observacionais, de reflexão, relatos de experiência, cartas ao editor, revisões sistemáticas, metanálises e integrativas de literatura; e estudos publicados entre dezembro de 2019 a julho de 2020. Foram excluídos estudos que não responderam à questão de pesquisa. 


\section{ARTIGO}

A busca dos artigos foi realizada de forma independente por dois pesquisadores. Primeiro foi feita a leitura dos títulos e resumos, com a seleção criteriosa dos artigos conforme os critérios de elegibilidade. Posteriormente, os trabalhos selecionados na etapa anterior foram lidos na íntegra. Por fim, esses estudos foram relidos e analisados conforme os critérios de elegibilidade, para só então, selecionar as publicações que compuseram a amostra final. Nesse processo de amostragem, os resultados dos dois

QUADRO 1 - Quantidade de artigos encontrados versus estratégias de busca. Cuité,

Paraíba, Brasil, 2020.

\begin{tabular}{|l|c|c|c|c|c|c|}
\hline \multicolumn{2}{|c|}{$1^{\circ}$ Etapa: Formulação do problema de pesquisa/questão norteadora (Quais medidas de prevenção } \\
da Covid-19 em pessoas que vivem com diabetes?) \\
\hline $\begin{array}{l}2^{\circ} \text { Etapa: Coleta de dados } \\
\text { (Definição das bases de } \\
\text { dados e busca de artigos) }\end{array}$ & \multicolumn{2}{|c|}{ Lilacs } & \multicolumn{2}{c|}{ Medline /Pubmed } & \multicolumn{2}{c|}{ Cinahl } \\
\cline { 2 - 7 } & Busca 1 & Busca 2 & Busca 1 & Busca 2 & Busca 1 & Busca 2 \\
\cline { 2 - 7 } & 07 & 07 & 29 & 306 & 40 & 47 \\
\hline $\begin{array}{l}3^{\circ} \text { Etapa: Avaliação dos } \\
\text { dados (Artigos } \\
\text { selecionados após leitura } \\
\text { de títulos e resumos) } \\
\text { *Aplicação dos filtros }\end{array}$ & 03 & 04 & 21 & 265 & 05 & 07 \\
\hline $\begin{array}{l}4^{\circ} \text { e 5 Etapas: Análise, } \\
\text { interpretação e } \\
\text { apresentação dos dados } \\
\text { (artigos selecionados } \\
\text { após leitura na íntegra) }\end{array}$ & 02 & 02 & 03 & 17 & 01 & 01 \\
\hline
\end{tabular}

Busca 1= Diabetes mellitus and coronavírus infections; Busca $2=$ Diabetes and covid-19

Para a coleta de dados dos artigos da amostra final, foi utilizado formulário adaptado a esta pesquisa contendo dados pesquisadores foram comparados $\mathrm{e}$ as diferenças solucionadas por consenso ou com a inclusão de um terceiro revisor, quando necessário, visando favorecer a validação da seleção dos estudos para análise.

A busca nas bases de dados resultou em 436 publicações, as quais foram selecionadas pelos critérios de elegibilidade, resultando na amostra final de 26 artigos, conforme é apontado no Quadro 1. 


\section{ARTIGO}

medidas de prevenção.

$\mathrm{Na}$ análise foram levantadas as medidas de prevenção da Covid-19 sugeridas nos artigos, sendo assim, os cuidados foram subdivididos em prevenção geral e prevenção específica para pessoas com diabetes, e apresentados quanto ao número quantitativo de citação e países de realização dos estudos.

Em relação ao nível de evidência, os estudos foram avaliados pela abordagem metodológica que foi baseada nas recomendações da Agency for Healthcare Research and Quality (AHRQ) ${ }^{(8)}$. Segundo sua classificação, a qualidade das evidências científicas estão assim categorizadas: nível 1 , metanálise de múltiplos ensaios clínicos controlados e randomizados; nível 2, estudo individual com delineamento experimental; nível 3, estudos quase experimentais; nível 4, estudos descritivos (não experimentais) ou abordagem qualitativa; nível 5 , relatos de caso ou experiência; nível 6 , opinião de especialistas.

\section{RESULTADOS}

No Quadro 2 é sumarizada a autoria, ano de publicação, nível de evidência, características metodológicas e objetivos dos estudos que compuseram esta revisão. Assim, observa-se uma predominância de estudos com nível de evidência 6,23 (88,5\%), desenvolvidos por pesquisadores de diferentes países, destacando-se a Índia 06 $(24,0 \%)$.

QUADRO 2- Síntese dos estudos de acordo com autoria, ano, nível de evidência, características metodológicas e objetivos. Cuité, Paraíba, Brasil, 2020.

\begin{tabular}{|l|l|}
\hline \multicolumn{1}{|c|}{$\begin{array}{c}\text { Autor/ano/nível de } \\
\text { evidência }\end{array}$} & \multicolumn{1}{|c|}{ Características metodológicas/objetivos } \\
\hline $\begin{array}{l}\text { Zuh L et al., 2020(9) } \\
\text { Nível evidência }=4\end{array}$ & $\begin{array}{l}\text { Estudo retrospectivo, multicêntrico, com 7.337 casos de Covid-19, } \\
\text { entre os quais 952 tinham diabetes tipo 2 pré-existente, realizado na } \\
\text { província de Hubei, China, com o objetivo verificar os níveis } \\
\text { plasmáticos de glicose e os resultados clínicos em pacientes com } \\
\text { diabetes tipo 2 e Covid-19. }\end{array}$ \\
\hline $\begin{array}{l}\text { Ministério da Saúde, } \\
2020^{(10)} \\
\text { Nível de evidência =6 }\end{array}$ & $\begin{array}{l}\text { Nota técnica de pesquisadores do Brasil com a finalidade de } \\
\text { orientar profissionais de saúde, gerentes de unidade e gestores sobre } \\
\text { os cuidados de pessoas com doenças crônicas na Atenção Primária à } \\
\text { Saúde perante a situação de pandemia de Covid-19. }\end{array}$ \\
\hline
\end{tabular}




\begin{tabular}{|c|c|}
\hline $\begin{array}{l}\text { Scott ES et al., } 2020^{(11)} \\
\text { Nível de evidência }=6\end{array}$ & $\begin{array}{l}\text { demonstrar a importância da continuidade dos cuidados de saúde } \\
\text { diabetes durante a pandemia de Covid-19. }\end{array}$ \\
\hline $\begin{array}{l}2020^{(12)} \\
\text { ncia }=6\end{array}$ & $\begin{array}{l}\text { esquisadores da Índia, com o objetivo de } \\
\text { continuidade dos cuidados de saúde em } \\
\text { de Covid-19. }\end{array}$ \\
\hline $\begin{array}{l}\text { l., } 2020^{(13)} \\
\text { ência }=6\end{array}$ & $\begin{array}{l}\text { ontando recomendações de prevenção da Covid-19 } \\
\text { abetes, divididas em: áreas específicas do diabetes, } 1 \\
\text { considerações logísticas. }\end{array}$ \\
\hline $\begin{array}{l}\text { odhary } \mathrm{J} \text { et al., } \\
\text { le evidência }=6\end{array}$ & $\begin{array}{l}\text { nvolvido por pesquisadores do Reino Unido, com o } \\
\text { monstrar atividades preventivas com relação a Covid- }\end{array}$ \\
\hline $\begin{array}{l}2020^{(15)} \\
\text { idência }=6\end{array}$ & $\begin{array}{l}\text { Editorial desenvolvido por pesquisadores da Coreia, com o objetivo de } \\
\text { fornecer recomendações para o tratamento do diabetes durante a } \\
\text { pandemia de Covid-19. }\end{array}$ \\
\hline $\begin{array}{l}\text { l., } 2020^{(16)} \\
\text { lência }=6\end{array}$ & $\begin{array}{l}\text { o objetivo de } \\
19 .\end{array}$ \\
\hline $\begin{array}{l}2020^{(17)} \\
\text { dência }=6\end{array}$ & $\begin{array}{l}\text { ndia, com o } \\
\text { tes mellitus, }\end{array}$ \\
\hline $\begin{array}{l}\text { eri } \mathrm{S} \text { et al., } \\
\text { e evidência }=6\end{array}$ & nedidas esp \\
\hline $\begin{array}{l}\text { Ovsky B et al., } \\
\text { 19) } \\
\text { de evidência }=6\end{array}$ & , com de \\
\hline $\begin{array}{l}2020^{(20)} \\
\text { idência }=6\end{array}$ & $\begin{array}{l}\text { fo de } \\
\text { foco }\end{array}$ \\
\hline $\begin{array}{l}2020^{(21)} \\
\text { lência }=6\end{array}$ & $\begin{array}{l}\text { tivo } \\
\text { mia }\end{array}$ \\
\hline $\begin{array}{l}\text { BA et al., } 2020^{(22)} \\
\text { de evidência }=6\end{array}$ & $\begin{array}{l}\text { torial desenvolvido por pesquisadores do Brasil com o obj } \\
\text { ntar a relação fisiopatológica da Covid-19 e do diabe }\end{array}$ \\
\hline $\begin{array}{l}\text { al., 2020(23) } \\
\text { dência }=6\end{array}$ & $\begin{array}{l}\text { om o } \\
\text { os de } \\
\text { s. }\end{array}$ \\
\hline$\frac{\text { Wicaksana }}{2020^{(24)}}$ AL et al., & $\begin{array}{l}\text { uisadores da Indonésia com o } \\
\text { gerenciamento do diabetes e } \\
\text { pandemia do Covid-19 para }\end{array}$ \\
\hline
\end{tabular}




\begin{tabular}{|c|c|}
\hline $\begin{array}{l}\text { Baneerj M et al., } \\
2020^{(25)} \\
\text { Nível de evidência }=6\end{array}$ & $\begin{array}{l}\text { Revisão narrativa desenvolvida por pesquisadores da Índia com o } \\
\text { objetivo de revisar os aspectos do atendimento centrado no paciente } \\
\text { por meio da educação em autogestão do diabetes na pandemia de } \\
\text { Covid-19. }\end{array}$ \\
\hline $\begin{array}{l}\text { Bornstein SR et al., } \\
2020^{(4)} \\
\text { Nível de evidência }=6\end{array}$ & $\begin{array}{l}\text { Editorial elaborado por pesquisadores da Alemanha, com o objetivo } \\
\text { de apresentar estratégias de manutenção do controle metabólico entre } \\
\text { os diabéticos durante a pandemia da Covid-19. }\end{array}$ \\
\hline $\begin{array}{l}\text { Tornese G et al., } \\
2020^{(26)} \\
\text { Nível evidência }=4\end{array}$ & $\begin{array}{l}\text { Estudo retrospectivo, desenvolvido com } 13 \text { indivíduos que viviam } \\
\text { com diabetes tipo } 1 \text {, na Itália, com o objetivo de verificar o impacto da } \\
\text { atividade física em casa no controle glicêmico, durante a quarente da } \\
\text { Covid-19. }\end{array}$ \\
\hline $\begin{array}{l}\text { Sinclair A et al., } 2020^{(27)} \\
\text { Nível de evidência }=6\end{array}$ & $\begin{array}{l}\text { Orientação emitida por pesquisadores do Reino Unido com o objetivo } \\
\text { de atender as necessidades especiais de residentes com diabetes em } \\
\text { casas de repouso durante a pandemia da Covid - } 19 \text {. }\end{array}$ \\
\hline $\begin{array}{l}\text { A, } 2020^{(28)} \\
\text { evidência }=6\end{array}$ & $\begin{array}{l}\text { Editorial desenvolvido por pesquisador dos Estados Unidos, } \\
\text { evidenciando a necessidade do controle da glicemia em pessoas com } \\
\text { diabetes como uma forma de prevenção de formas graves da Covid- } \\
19 \text {. }\end{array}$ \\
\hline $\begin{array}{l}\text { A et al., } 2020^{(29)} \\
\text { de evidência }=1\end{array}$ & $\begin{array}{l}\text { Meta-análise desenvolvida por pesquisadores da Índia, com o objetivo } \\
\text { de apontar a gravidade da Covid-19 em pacientes com diabetes. }\end{array}$ \\
\hline $\begin{array}{l}\text { Katulanda P et al., } \\
2020^{(30)} \\
\text { Nível de evidência }=6\end{array}$ & $\begin{array}{l}\text { Revisão da literatura realizada por pesquisadores do Sri Lanka com o } \\
\text { objetivo de apontar os desafios percebidos para prevenção e manejo } \\
\text { da Covid-19 em pessoas com diabetes. }\end{array}$ \\
\hline $\begin{array}{l}\text { Ministério da Saúde, } \\
2020^{(31)} \\
\text { Nível de evidência }=6\end{array}$ & $\begin{array}{l}\text { Recomendações elaboradas por pesquisadores de El Salvador com o } \\
\text { objetivo de apontar orientações sobre como lidar com a Covid-19 em } \\
\text { pessoas com diabetes mellitus e outras doenças crônicas. }\end{array}$ \\
\hline $\begin{array}{l}\text { Balducci } \quad \text { S et al., } \\
2020^{(32)} \\
\text { Nível de evidência }=6\end{array}$ & $\begin{array}{l}\text { Editorial desenvolvido por pesquisadores da Itália apontando a } \\
\text { atividade física como medica necessária para o bom controle } \\
\text { glicêmico durante a pandemia da Covid-19, atuando portanto, na } \\
\text { prevenção. }\end{array}$ \\
\hline $\begin{array}{l}\text { Taminato M et al., } \\
2020^{(33)} \\
\text { Nível de evidência }=6\end{array}$ & $\begin{array}{l}\text { Revisão da literatura desenvolvida por pesquisadores do Brasil com o } \\
\text { objetivo de identificar evidências científicas disponíveis sobre a } \\
\text { eficácia e a segurança das máscaras de tecido para a comunidade. }\end{array}$ \\
\hline & $\begin{array}{l}\text { medidas de prevenção da Covid-19 } \\
\text { sugeridas nos artigos, sendo subdivididas em } \\
\text { prevenção geral e prevenção específica para }\end{array}$ \\
\hline
\end{tabular}

No Quadro 3 são apresentadas as

QUADRO 3- Descrição das medidas de prevenção da Covid-19, número de artigos que apresentaram a citação e países de realização dos estudos. Cuité, Paraíba, Brasil, 2020. 
ARTIGO

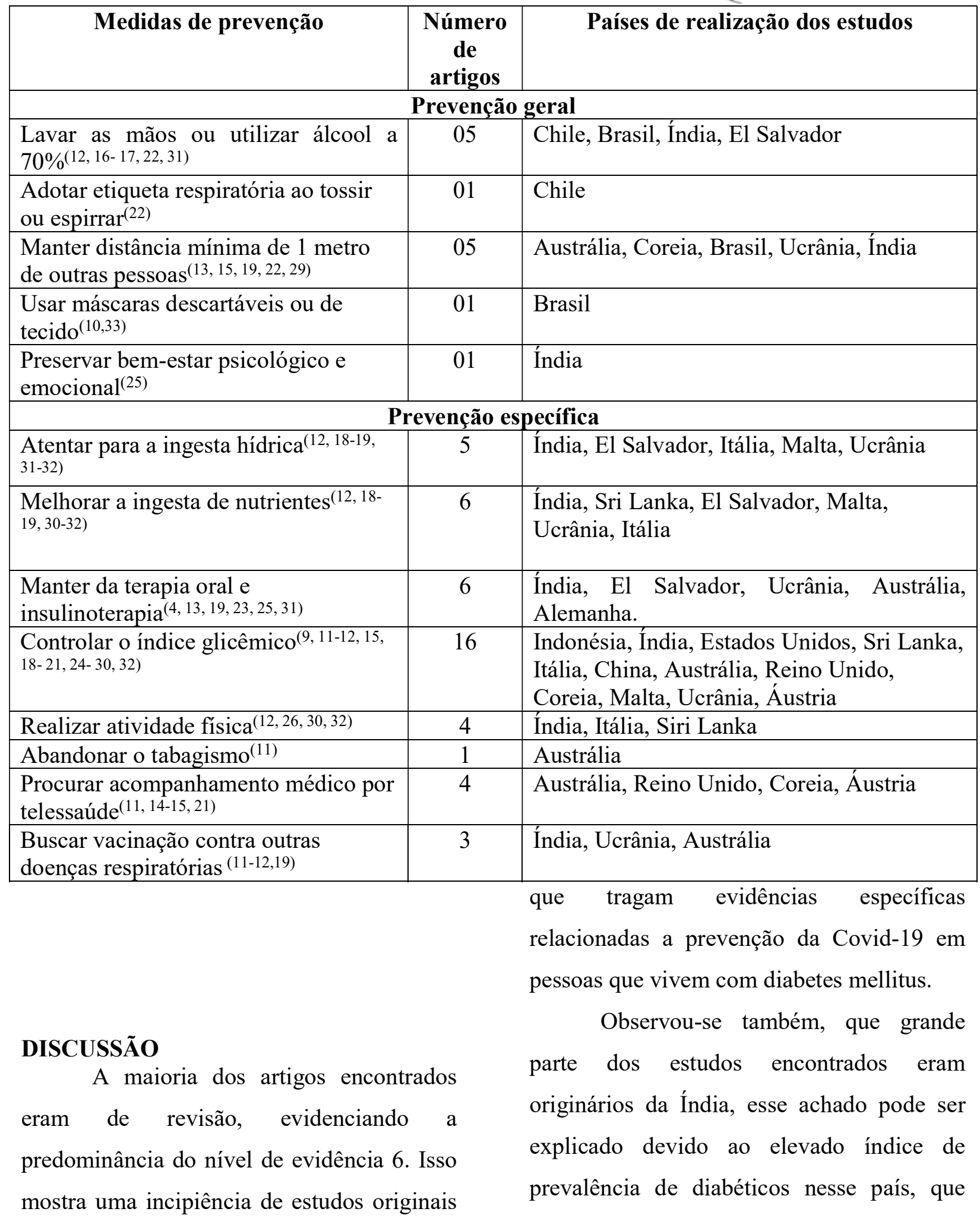




\section{ARTIGO}

vive uma transição epidemiológica, no sentido de apresentar uma elevação das doenças crônicas não transmissíveis mais acelerada $^{(34)}$.

As medidas preventivas encontradas, foram divididas, nesse artigo, em duas categorias, sendo elas: prevenção geral e prevenção específica. $\mathrm{Na}$ categoria prevenção geral, são descritos os cuidados comuns a qualquer pessoa, independentemente de o indivíduo apresentar diabetes mellitus ou não. Sendo assim, de acordo com os achados, foram evidenciados como cuidados gerais: lavar as mãos ou utilizar álcool a 70\%, adotar etiqueta respiratória ao tossir ou espirrar, manter distância mínima de um metro de outras pessoas, usar máscaras descartáveis ou de tecido e preservar bem estar psicológico e emocional.

A lavagem das mãos é um importante mecanismo para remoção de suor, sujidade, oleosidade, células mortas e microorganismos. Sabe-se que as mãos abrigam uma quantidade considerável de patógenos de baixa a alta virulência, sendo um meio comum de contaminação cruzada. Deste modo, a higienização mecânica com água e sabão é ideal para a diminuição da propagação de doenças infecciosas ${ }^{(35)}$. Da mesma forma, as soluções alcóolicas a 70\%, também desempenham ótima atividade da remoção de microorganismos, e podem ser utilizadas principalmente em locais onde não há possibilidade de lavar as mãos imediatamente. Porém, ressalta-se que a utilização dessa substância não substitui a lavagem das mãos, principalmente se houver sujidade $^{(36)}$.

A utilização de máscaras é recomendada principalmente em locais onde há fluxo de pessoas, evitando o contato das vias aéreas com partículas contaminadas pelo vírus ${ }^{(37)}$. No que diz respeito a produção de máscaras caseiras, investigação aponta que sua indicação consiste em uma medida social de baixo custo para a população geral, especialmente para o momento em que questões sobre a transmissibilidade de assintomáticos e oligossintomáticos não estão totalmente definidas, bem como uma intervenção para os países pouco desenvolvidos e em desenvolvimento ${ }^{(33)}$.

Destaca-se também a recomendação de etiqueta respiratória ao tossir ou espirrar, que consiste em colocar lenços e/ou antebraço na boca e nariz para não espalhar gotículas no ambiente, e evitar espalhar os microorganismos nas mãos ${ }^{(38)}$. Ademais, o distanciamento social se mostra uma medida 


\section{ARTIGO}

de prevenção efetiva para conter a disseminação do vírus, visto que a doença em questão é transmitida através do contato muito próximo entre as pessoas. $\mathrm{O}$ distanciamento consiste em evitar interações físicas entre as pessoas de uma comunidade, evitando que portadores do vírus ainda não identificados contaminem outras pessoas ${ }^{(39)}$.

Pesquisa $^{(40)}$ apontou que a combinação de uso de máscaras, mesmo que assintomáticos, e distanciamento social foi a principal responsável pela supressão no surto de casos em Wuhan. Portanto, independente da máscara utilizada, no que diz respeito a eficiência da filtragem, surtirá efeito, quando utilizada associada as outras medidas, como distanciamento social, boas práticas de etiqueta respiratória e higienização regular das mãos ${ }^{(33)}$.

Tratando-se do bem-estar psicológico e emocional, vê-se as emoções e o estado psicológico como questões que implicam na saúde e qualidade de vida dos indivíduos. Isso é explicado, pois quando não se há um equilíbrio emocional, pode-se ocorrer uma somatização de sentimentos e adoecimento. Além disso, emoções negativas são capazes de induzir os indivíduos a reduzir hábitos saudáveis relacionados a atividade física, dieta, descanso, piorando ainda mais o quadro de saúde ${ }^{(41)}$.

$\mathrm{Na}$ categoria prevenção específica, relacionado aos cuidados com os pacientes que vivem com diabetes mellitus, diversos estudos enfatizaram a importância de atenção para a ingesta hídrica, melhorar ingesta de nutrientes, manutenção da terapia oral e insulinoterapia, controlar o índice glicêmico, realizar atividade física, abandonar o tabagismo, procurar acompanhamento médico por telessaúde e buscar vacinação contra outras doenças respiratórias.

No que diz respeito a ingesta hídrica, aponta-se que as pessoas que vivem com diabetes possuem grande risco de desidratação, devido altas concentrações de glicose no sangue, o que exige maior trabalho do sistema renal em eliminá-la, levando consigo considerável quantidade de água, nisso, entende-se que a ingesta hídrica é extremamente importante principalmente para melhora da imunidade, como também para diminuição do risco de agravamento do quadro, uma vez que um dos sintomas da Covid-19 é justamente a desidratação(42).

Em se tratando da melhoria da ingesta de nutrientes, estudo aponta a necessidade da ingestão apropriada de 


\section{ARTIGO}

proteínas, como também estar atento a deficiências de minerais e vitaminas ${ }^{(19)}$. Para tanto, a alimentação saudável possibilita o bem-estar das funções orgânicas, e é comprovado que a adequação nutricional promove a redução de $1 \%$ a $2 \%$ nos níveis de hemoglobina glicada, independentemente do tipo de diabetes e tempo de diagnóstico, devendo ser estabelecida de forma individualizada, propondo as adequações de macro e micronutrientes necessários para $\mathrm{o}$ efetivo controle glicêmico ${ }^{(43)}$.

A prática de exercícios físicos reduz a incidência e a duração de infecções do trato respiratório superior, além de ser preditora para benefícios na saúde, por acelerar as adaptações metabólicas e hormonais, causando diminuição do colesterol e da necessidade de insulina; promover a melhora cardiorrespiratória e a prevenção de complicações secundárias do diabetes $^{(44)}$.

Em uma opinião de especialistas voltada para idosos, evidenciou-se que a inatividade física surgiu como um efeito adverso das medidas de isolamento social adotadas para combater a disseminação da Covid-19, assim, os pesquisadores propõem a implementação de programas de exercícios em casa, que são relevantes para manutenção de massa muscular e prevenção/controle de outras morbidades, como o diabetes ${ }^{(45)}$.

Concernente a terapia oral e insulinoterapia, recomenda-se a manutenção do tratamento, no sentido de gerenciar o diabetes para uma possível prevenção com relação a complicações advindas da Covid19(30). Em estudo $^{(12)}$, sugere-se a insulinoterapia como uma medida a ser utilizada preferencialmente, caso indivíduo hospitalizado apresente hiperglicemia. É sabido também, que os usuários com diabetes tiveram dificuldade de acesso aos insumos para manutenção do tratamento, devido a sua falta em farmácias e ao medo de sair de casa ${ }^{(13)}$.

Investigação $^{(11)}$ também recomendou a cessação do tabagismo, visto que este tem associação com o risco elevado de desenvolver problemas pulmonares, de doença cardiovascular e de acidente vascular cerebral em pacientes diabéticos, além de aumentar o risco de início e a progressão da nefropatia, uma vez que está associado à diminuição da taxa de filtração glomerular. Ademais, pacientes diabéticos fumantes também apresentam pior controle glicêmico e maior risco de hipoglicemia ${ }^{(46)}$.

$$
\text { O controle glicêmico, é o }
$$
ponto chave para a manutenção dos cuidados 


\section{ARTIGO}

em pessoas que vivem com diabetes, visto que alterações na glicemia, sobretudo a hiperglicemia, promovem distúrbios fisiológicos na cicatrização, complicações vasculares, neuropáticas e à inibição do sistema de defesa, expondo-os de maneira mais agressiva ao risco de complicações inerentes a infecção da Covid-19(9,11-12, 30,32).

No entanto, pontua-se que o momento de isolamento social durante a pandemia de Covid-19, pode influenciar na manutenção desse biomarcador. Conforme demonstram dados de estudo desenvolvido na China, que evidenciou que idosos com diabetes mellitus tipo 2 experimentaram piora do controle glicêmico, evidenciando impacto do distanciamento social no estilo de vida, devido à limitação de atividades físicas, a restrição no suprimento de alimentos e consequente alteração nos hábitos alimentares, a dificuldade na aquisição de medicamentos antidiabéticos e a falta de acompanhamento médico para manutenção de uma rotina clínica ${ }^{(47)}$.

Ademais, estudos ${ }^{(12,19)}$ sugerem que as pessoas com diabetes busquem vacinação contra a influenza e a pneumonia, pois essa imunização pode reduzir as chances de pneumonia bacteriana secundária após infecção viral do trato respiratório. Destaca- se que na crise sanitária diante da pandemia da Covid-19, a adesão à campanha de vacinação como medida de prevenção, não só diminui a incidência de casos de influenza e pneumonia, como também viabilizam o diagnóstico mais preciso da Covid-19(48).

Por fim, o uso de tecnologias para o acompanhamento remoto e a consulta por telessaúde tornaram-se um meio bastante difundido, e não obstante, sabe-se que são estratégias úteis para que as pessoas tenham acesso a orientações para promoção e prevenção em saúde, sobretudo as pessoas que vivem com doenças crônicas como o diabetes $^{(11,14-15,21)}$. No entanto, a utilização de tais recursos exige aceitação dos pacientes, treinamento e disponibilidade dos meios tecnológicos necessários ${ }^{(49)}$.

Corroborando os dados apresentados, investigação desenvolvida com 1.700 brasileiros, mostrou os impactos da Covid19 em pessoas com diabetes, sugerindo a mudança de hábitos, como por exemplo: $95,1 \%$ dos entrevistados reduziram sua frequência de sair de casa, entre os que monitoraram a glicose no sangue em casa durante a pandemia (91,5\%), a maioria $(59,4 \%)$ apresentou aumento, diminuição ou maior variabilidade nos níveis de glicose, $38,4 \%$ adiaram suas consultas 


\section{ARTIGO}

médicas e/ou exames de rotina, e 59,5\% reduziram a atividade física ${ }^{(50)}$. Portanto, observa-se a relevância e contribuição dos achados desta pesquisa, visto que nortearão as práticas de prevenção e controle, instrumentalizando um cuidado baseado em fundamentos teórico-científicos, a fim de minimizar os impactos da Covid-19 na vida de usuários diabéticos.

Quanto as limitações desse estudo, destaca-se uma amostra elevada de estudos com nível de evidência igual a 6 , o que desperta a necessidade do desenvolvimento de mais investigações, principalmente de caráter observacional, para a evidências de dados mais precisos e representativos.

\section{CONCLUSÃO}

Os achados extraídos dos artigos levaram a construção de duas categorias envolvendo medidas preventivas, sendo elas: prevenção geral e prevenção específica. $\mathrm{Na}$ categoria prevenção geral, foram descritos os cuidados comuns a qualquer pessoa, independentemente de o indivíduo apresentar diabetes mellitus ou não. $\mathrm{Na}$ categoria prevenção específica, foram traçadas intervenções com o objetivo de manutenção da imunidade das pessoas que viviam com diabetes.

\section{REFERÊNCIAS}

1. Guan W, Ni Z, Hu Y, Liang W, Ou C Clinical Characteristics of Coronavirus Disease 2019 in China. N Engl J Med. [internet]. 2020 [acesso em: 01 jun 2020]; 382: 1708-1720. Disponível em: http://dx.doi.org/10.1056/NEJMoa2002032.

2. Huang I, Lim MA, Pranata R. Diabetes mellitus is associated with increased mortality and severity of disease in COVID19 pneumonia e A systematic review, etaanalysis, and meta-regression. Diabetes \& Metabolic Syndrome: Clinical Research \& Reviews. [internet]. 2020 [acesso em: 01 jun 2020]; 14(4): 395-403. Disponível em: https://doi.org/10.1016/j.dsx.2020.04.018.

3 . Sociedade Brasileira de diabetes. Notas de esclarecimentos da Sociedade Brasileira de Diabetes sobre o coronavírus (COVID-19). [internet]. 2020 [acesso em: 19 julho de 2020]. Disponível em: https://www.diabetes.org.br/covid-19/notasde-esclarecimentos-da-sociedade-brasileirade-diabetes-sobre-o-coronavirus-covid-19/.

4. Bornstein SR, Dalan R, Hopkins D, Mingrone G, Boehm BO. Endocrine and metabolic link to coronavirus infection. Nature Reviews Endocrinology. [internet]. 2020 [acesso em: 10 jul 2020]; 16: 297-298. Disponível em: https://doi.org/10.1038/ s41574-020-0353-9.

5. Hussain A, Bhowmik B, Moreira NCV. COVID-19 and diabetes: Knowledge in progress. Elsevier: Diabetes Research and Clinical Practice. [internet]. 2020 [acesso em: 11 mai 2020]; 162. Disponível em: https://doi.org/10.1016/j.diabres.2020.10814 2.

6. Oliveira AC, Lucas TC, Iquiapaza RA. O que a pandemia da Covid-19 tem nos ensinado sobre adoção de medidas de precaução? Texto Contexto Enferm. [internet]. 2020 [acesso em: 26 mai 2020]; 


\section{ARTIGO}

29.

Disponível

https://doi.org/10.1590/1980-265X-TCE2020-0106.

7. Whittemore R, Knafl K. The integrative review: update methodology. J Adv Nurs. [internet]. 2005 [acesso em: 26 mai 2020]; 52(5): 546-53. Disponível em: https://doi.org/10.1111/j.1365-

2648.2005.03621.x.

8. Stetler CB, Morsi D, Rucki S, Broughton S, Corrigan B, Fitsgerald J, et al. Utilizationfocused integrative reviews in a nursing service. Appl Nurs Res. [internet]. 1998 [acesso em: 26 mai 2020]; 11(4):195-206. Disponível em: https://doi.org/10.1016/S08971897(98)80329-7.

9. Zhu L, She ZG, Cheng X, Qin JJ, Zhang XJ. Association of Blood Glucose Control and Outcomes in Patients with COVID-19 and Pre-existing Type 2 Diabetes. Cell Metabolism. [internet]. 2020 [acesso em: 20 jun 2020]; 31(6): 1068-1077. Disponível em: https://www.sciencedirect.com/science/articl e/pii/S1550413120302382.

10. Ministério da Saúde. Atenção a pessoas com doenças crônicas na APS diante da situação de pandemia de Covid-19. [internet]. 2020 [acesso em: 20 jun 2020]. Disponível

em: https://pesquisa.bvsalud.org/portal/reso urce/pt/biblio-1096507.

11. Scott ES, Jenkins AJ, Fulcher GR. Challenges of diabetes management during the COVID-19 pandemic. Med J Aust. [internet]. 2020 [acesso em: 21 jun 2020]; 213(2): 56-57. Disponível em: https://onlinelibrary.wiley.com/doi/10.5694/ mja2.50665.

12. Gupta R, Ghosh A, Singh AK, Misra A. Clinical considerations for patients with diabetes in times of COVID-19 epidemic. Diabetes y Metabolic Syndrome. [internet]. 2020 [acesso em: 22 jun 2020]; 14(3) 211-
$212 . \quad$ Disponível em: https://pubmed.ncbi.nlm.nih.gov/32172175/. 13. Sacks LJ, Phama CT, Fleming N, Neohab SL, Ekinci EI. Considerations for people with diabetes during the Coronavirus Disease (COVID-19) pandemic. Diabetes Research and Clinical Practice. [internet]. 2020 [acesso em: 24 jun 2020]; 166. Disponível em: https://www.sciencedirect.com/science/articl e/pii/S0168822720305489.

14. Seewoodhary, J, Oozageer, R. Coronavirus and diabetes: an update. Pract Diab. [internet]. 2020 [acesso em: 26 jun 2020]; 37: 41-42. Disponível em: https://onlinelibrary.wiley.com/doi/10.1002/ pdi. 2260 .

15. Noh J, Jeong IK, Chung DR, Yoon KH. Coronavirus Disease 2019 and Diabetes: The Epidemic and the Korean Diabetes Association Perspective. Diabetes \& Metabouilsm Journal. [internet]. 2020 [acesso em: 24 jun 2020]; 44(3): 372. Disponível em: https://www.researchgate.net/publication/34 2596732 Coronavirus Disease 2019 and Diabetes The Epidemic and the Korean Diabetes Association Perspective. 16. Bravo FP, G CL, Chacón GC, Gatica JD. Coronavirus y diabetes. Revista Chilena de Endocrinología y Diabetes. [internet]. 2020 [acesso em: 31 jun 2020]; 13(2): 37-42. Disponível em: http://revistasoched.cl/2_2020/0.pdf.

17. Pal R, Bhadada SK. COVID-19 and diabetes mellitus: an unholy interaction of two pandemics. Diabetes \& Metabolic Syndrome: Clinical Research \& Reviews. [internet]. 2020 [acesso em: 20 jun 2020]; 14(4): 513-517. Disponível em: https://www.sciencedirect.com/science/articl e/pii/S1871402120301144.

18. Cuschieri S, Grech S. COVID-19 and diabetes: The why, the what and the how. J 
Diabetes Complications. [internet]. 2020 [acesso em: 01 jul 2020]; 22. Disponível em: https://www.ncbi.nlm.nih.gov/pmc/articles/P MC7242955/.

19. Mankovsky B, Halushko Ó. Covid-19 in diabetes patients in ukraine: lessons for doctors and patients. Georgian Med News. [internet]. 2020 [acesso em: 10 jul 2020]; 300: 0105-112. Disponível em: https://pesquisa.bvsalud.org/globalliterature-on-novel-coronavirus-2019ncov/resource/en/covidwhomdl-32535573. 20. Pal R, Bhansali A. COVID-19, diabetes mellitus and ACE2: The conundrum. Diabetes Res Clin Pract. [internet] 2020 [acesso em: 10 jul 2020]; 162. Disponível em:

https://www.ncbi.nlm.nih.gov/pmc/articles/P MC7118535/.

21. Peric S, Stulnig TM. Diabetes and COVID-19: Disease-Management-People. Wien Klin Wochenschr. [internet]. 2020 [acesso em: 20 jul 2020]; 132(13-14): 35661 . Disponível em:

https://pesquisa.bvsalud.org/globalliterature-on-novel-coronavirus-2019ncov/resource/en/covidwho-327003.

22. Pitito BA, Ferreira FSG. Diabetes e covid-19: mais do que a soma de duas morbidades. Revista de Saúde Pública. [internet]. 2020 [acesso em: 10 jul 2020]; 54(54). Disponível em: https://www.scielo.br/pdf/rsp/v54/15188787-rsp-54-54.pdf.

23. Singh AK, Gupta R, Ghosh A, Misra A. Diabetes in COVID-19: Prevalence, pathophysiology, prognosis and practical considerations. Diabetes Metab Syndr. [internet]. 2020 [acesso em 19 jul 2020]; 14(4): 303-310. Disponível em: https://www.sciencedirect.com/science/articl e/pii/S1871402120300631.

24. Wicaksana AL, Hertanti NS, Ferdiana A, Pramono RB. Diabetes management and specific considerations for patients with diabetes during coronavirus diseases pandemic: A scoping review. Diabetes \& Metabolic Syndrome: Clinical Research \& Reviews. [internet]. 2020 [acesso em: 15 jul. 2020]; 14(5): 1109-1120. Disponível em: https://www.sciencedirect.com/science/articl e/pii/S187140212030240X?dgcid=rss_sd_all 25. Banerjee M, Chakraborty S; Pal R. Diabetes self-management amid COVID-19 pandemic. Diabetes Metabolic Syndrome. [internet]. 2020 [acesso em: 20 jun 2020]; 14(4): 351-354. Disponível em: https://pesquisa.bvsalud.org/controlecancer/r esource/pt/mdl-32311652.

26. Tornese G, Ceconi V, Monasta L, Carletti C, Faleschini E, Barbi E. Glycemic Control in Type 1 Diabetes Mellitus During COVID-19 Quarantine and the Role of InHome Physical Activity. Diabetes Technology \& Therapeutics. [internet]. 2020 [acesso em: 21 jun 2020]; 22(6). Disponível em:

https://www.liebertpub.com/doi/10.1089/dia. 2020.0169.

27. Sinclair A, Dathariya K, Burr O, Nagi D, Higgins K, Hopikns D, et al. Guidelines for the management of diabetes in care homes during the Covid-19 pandemic. Diabetic Medicine. [internet]. 2020 [acesso em: 20 jun 2020]; 37: 1090-1093. Disponível em: https://onlinelibrary.wiley.com/doi/epdf/10.1 111/dme.14317.

28. Brusfky A. Hyperglycemia, hydroxychloroquine, and the COVID-19 pandemic. Journal Medical of Virology. [internet]. 2020 [acesso em: 15 jul 2020]; 92: 770-775. Disponível em: https://onlinelibrary.wiley.com/doi/epdf/10.1 002/jmv.25887.

29. Kumar A, Arora A, Sharma P, Anikhindi SA, Bansal N. Is diabetes mellitus associated with mortality and severity of COVID-19? A meta-analysis. Diabetes y Metabolic 
Syndrome. [internet]. 2020 [acesso em: 24 jun 2020]; 14(4): 535-545. Disnponível em: https://www.ncbi.nlm.nih.gov/pmc/articles/P MC7200339/.

30. Katulanda P, Dissanayake HA, Ranathunga I, Ratnasamy V, Wijewickrama PSA, Yogendranathan D, et al. Prevention and management of COVID-19 among patients with diabetes: an appraisal of the literature. Diabetologia. [internet]. 2020 [acesso em: 17 jul 2020]; 63(8): 1440-52. Disponível em: https://link.springer.com/article/10.1007/s00 125-020-05164-x.

31. Bendek ACO, Montoya FJA, Cardoza CGA. Recomendaciones pala el abordaje integral por la enfermidade COVID-19 em personas com enfermidades crônicas no transmisibles y personas adultas mayores. Ministério de Salud. [internet]. 2020 [acesso em 13 jul 2020]. Disponível em: https://pesquisa.bvsalud.org/controlecancer/r esource/pt/biblio-1087708.

32. Balducci S, Coccia EM. Sedentariness and physical activity in type 2 diabetes during the COVID-19 pandemic. Diabetes Metabolism Research and Reviews. [internet]. 2020 [acesso em: 13 jul 2020]. Disponível em: https://onlinelibrary.wiley.com/doi/epdf/10.1 002/dmrr.3378.

33. Taminato $\mathrm{M}$, Mizusaki-Imoto $\mathrm{A}$, Saconato H, Franco ES, Puga ME, Duarte $\mathrm{ML}$, et al. Máscaras de tecido na contenção de gotículas respiratórias - revisão sistemática. Acta Paul Enferm. [internet] 2020 [acesso em: 20 jul 2020]:eAPE20200103. Disponível em: http://dx.doi.org/10.37689/actaape/2020AR0103.

34. Geltsedzer P, Manne-Goehler J, Theilmann M. Diabetes e hipertensão na Índia: Um estudo nacionalmente representativo de 1,3 milhão de adultos.
JAMA Intern Med. [internet]. 2018 [acesso em: 25 jul 2020]; 178(3): 363-372. Disponível em: https://jamanetwork.com/journals/jamaintern almedicine/article-abstract/2670035.

35. Benevides JC, Pedroni VT, Carvalho ACG. Conhecendo a higienização das mãos, para uma assistência qualificada. Revista Interdisciplinar do Pensamento Científico. 2019 [acesso em: 10 jul 2020]; 5(70). Disponível em: http://reinpec.org/reinpec/index.php/reinpec/ article/view/434/355.

36. Sequinel R, Lenz GF, Silva JFLB, Silva FR. Soluções a base de álcool para higienização das mãos e superfícies na prevenção da covid-19: compêndio informativo sob o ponto de vista da química envolvida. Química Nova. 2020 [acesso em: 10 jul 2020]; 43(5): 679-684. Disponível em: https://www.scielo.br/pdf/qn/v43n5/01004042-qn-43-05-0679.pdf.

37. Abud CO, Souza LP. Uso obrigatório de máscara facial para conter a COVID-19 no Brasil: limitação legítima ao direito fundamental de autodeterminação. Vigilância Sanitária em Debate. 2020 [acesso em: 20 jul 2020]. Disponível em: https://visaemdebate.incqs.fiocruz.br/index.p $\mathrm{hp} /$ visaemdebate/article/view/1651/1193.

38. Freitas MGG. COVID-19: Procedimentos em estabelecimentos de restauração e bebidas. Orientação $n^{\circ}$ 023/2020 de 08/05/2020. [acesso em: 20 jul 2020]. Disponível em: https://santaluzia.com/dgs.pdf.

39. Aquino EML, Silveira IH, Pescarini JM, Aquino R, Souza-Filho JA. Medidas de distanciamento social no controle da pandemia de COVID-19: potenciais impactos e desafios no Brasil. Ciência \& Saúde Coletiva. [internet]. 2020 [acesso em: 21 jul 2020]; 25: 2423-2446. Disponível em: https://www.scielosp.org/pdf/csc/2020.v25su 
ppl1/2423-2446/pt.

40. Garcia LP. Uso de máscara facial para limitar a transmissão da COVID-19. Epidemiol. Serv. Saúde. [internet]. 2020 [acesso em: 22 jul 2020]; 29(2). Disponível em:

https://www.scielosp.org/article/ress/2020.v 29n2/e2020023/pt/.

41. Martins MCA, Melo JMCD. Emoção...emoções... que implicações para a saúde e qualidade de vida? Millenium Journal of Education, Technologies and Health. 2016 [acesso em: 20 jul 2020]; 34(13). Disponível em: https://revistas.rcaap.pt/millenium/article/vie w/8362.

42. Davies MJ, D'Alessio DA, Fradkin J, Kernan WN, Mathieu C, Mingrone G, et al. Management of Hyperglycemia in Type 2 Diabetes, 2018. A Consensus Report by the American Diabetes Association (ADA) and the European Association for the Study of Diabetes (EASD). Diabetes Care. [internet]. 2018 [acesso em: 23 jul 2020]; 41(12). Disponível em: https:/care.diabetesjournals.org/content/earl y/2018/09/27/dci18-0033.full-text.pdf.

43. Fernandez NM, Cazelli C, Teixeira, RJ. Gerenciamento do controle glicêmico do diabetes mellitus tipo 2 na Estratégia de Saúde da Família. Revista HUPE. [internet]. 2016 [acesso em: 19 jul 2020]; 15(03). Disponível em: https://www.epublicacoes.uerj.br/index.php/revistahupe/art icle/view/29447.

44. Marçal DFS, Alexandrino EG, Cortez LER, Bennemann RM. Effects of physical exercise on type 1diabetes mellitus: a systematic review of clinical and randomized tests. J. Phys. Educ. [internet]. 2018 [acesso em: 19 jul 2020]; 29. Disponível https://www.scielo.br/pdf/jpe/v29/2448-

2455-jpe-29-e2917.pdf.

45. Roschel H, Artioli GG, Gualano B. Risk of Increased Physical Inactivity During COVID -19 Outbreak in Older People: A Call for Actions. Journal of the American Geriatrics Society [internet]. 2020 [acesso em: 26 jul 2020]; 68(6). Disponível em: https://doi.org/10.1111/jgs.16550.

46. Zhu P, Pan, X, Sheng L, Chen, H, Pan, A. Cigarette Smoking, Diabetes, and Diabetes Complications: Call for Urgent Action. Curr Diab Rep. [internet]. 2017 [acesso em: 21 jul 2020]; 17(9): 78. Disponível em: https://pubmed.ncbi.nlm.nih.gov/28766247/. 47. Xue T, Li Q, Zhang Q, Lin W, Wen J, Li $\mathrm{L}$, et al. Blood glucose levels in elderly subjects with type 2 diabetes during COVID-19 outbreak: a retrospective study in a single center. Medrxiv: Endocrinology. [internet] 2020 [acesso em: 16 jun 2020]. Disponível

em: http://medrxiv.org/lookup/doi/10.1101/2020. 03.31.20048579.

48. Lana RM, Coelho FC, Gomes MFC, Cruz OG, Bastos LS, Maciel DA. The novel coronavirus (SARS-CoV-2) emergency and the role of timely and effective national health surveillance. Cad. Saúde Pública [Internet]. 2020 [acesso em: 26 jul 2020]; 36(3): e00019620. Disponível em: http://www.scielo.br/scielo.php?script=sci_a rttext\&pid=S0102311X2020000300301\&lng=en.

49. López-Torres J, Rabanales J, Fernández $\mathrm{R}$, López FJ, Llanos P, Victoria R. Resultados de un programa de telemedicina para pacientes con diabetes tipo 2 en atención primaria. Gac Sanit [Internet]. 2015 [acesso em: 26 jul 2020]; 29(1): 55-58. Disponível em: http://scielo.isciii.es/scielo.php?script=sci ar ttext\&pid=S0213- 
91112015000100010\&lng=pt. http://dx.doi. org/10.1016/j.gaceta.2014.08.003.

50. Barone MTU, Harnik SB, Luca PV, Lima BLS, Wieselberg RJP, Ngongo B, The impact of COVID-19 on people with diabetes in Brazil. Diabetes Researchand Clinical Practice. [internet] 2020 [acesso em 26 jul 2020]; I66: I08304. Disponível em: https://doi.org/10.1016/j.diabres.2020.10830 4

Recebido: 2020-07-27

Aceito: 2020-08-10 